\title{
Rationality, monotonicity and consistency in cooperative games: some (im)possibility results
}

\author{
Pedro Calleja and Francesc Llerena *
}

\begin{abstract}
On the domain of cooperative transferable utility games, we investigate if there are single valued solutions that reconcile rationality, consistency and monotonicity (with respect to the worth of the grand coalition) properties. This paper collects some impossibility results on the combination of core selection with either complement or projected consistency, and core selection, max consistency and monotonicity. By contrast, possibility results show up when combining individual rationality, projected consistency and monotonicity.
\end{abstract}

\section{Introduction}

A transferable utility coalitional game is specified by a finite set of players and a realvalued function defined on all coalition of players. A (single-valued) solution is a function which assigns for each game an efficient payoff vector. The question is whether efficiency (or Pareto-optimality) can be combined with suitable properties. This is one of the objectives of the axiomatic approach: to identify desirable properties of solutions and explore the compatibility among them.

The core (Gillies, 1953) of a game is the set of feasible outcomes that can not be improved upon by any coalition of players. A solution satisfies core selection if it selects a core element for any game with a non-empty core. Individual rationality imposes that every player gets at least his individual worth whenever it is possible. These two properties are requirements of rationality for solutions and particularly attractive for cooperation.

Another important property frequently used to characterize solutions is consistency. A solution satisfies consistency if it makes coherent choices in both the original game and the reduced game. Depending on how reduced games are defined, several notions of consistency are obtained. Here we consider max consistency (Davis and Maschler, 1965), complement consistency (Moulin, 1985) and projected consistency (Funaki, 1998). ${ }^{1}$

In combining properties of rationality with properties of consistency we find some drawbacks. Hwang (2013) shows that individual rationality is incompatible with either

\footnotetext{
* Dep. de Matemàtica Econòmica, Financera i Actuarial, Universitat de Barcelona Dep. de Gestió d'Empreses, Universitat Rovira i Virgili-CREIP, e-mail: calleja@ub.edu (Pedro Calleja), francesc.llerena@urv.cat (Francesc Llerena).

${ }^{1}$ For comprehensive surveys on the consistency principles, the reader is referred to Driessen (1991) and Thomson (2003) .
} 
complement or max consistency. On the other hand, although the core itself obeys the above three consistency properties, it turns out that core selection is neither compatible with complement consistency nor with projected consistency. Remarkably, individual rationality and core selection together are incompatible with any of these consistency properties. By constrast, the prenucleolus (Schmeidler, 1969) satisfies core selection and max consistency, and the center of imputations (Driessen and Funaki, 1991) individual rationality and projected consistency.

Aggregate monotonicity (Megiddo, 1974) says that if the worth of the grand coalition increases, whereas the worth of other coalitions remain unchanged, then everyone's payoff should weakly increase. Most of solutions that are aggregate monotonic, like the Shapley value (Shapley, 1953), the per-capita prenucleolus (Grotte, 1970) or the center of imputations, satisfy equal surplus division, which implies that any variation of the worth of the grand coalition is distributed equally among all players. Capturing the idea that players may commit in distributing monotonically, but not equally, variations in their wealth, we introduce regular aggregate monotonicity. The center of imputations, which has been recently axiomatized in Béal et al. (2014), Casajus and Huettner (2014), Chun and Park (2012) and van den Brink (2007) without making use of monotonicity properties, comes out to be the unique single-valued solution satisfying individual rationality and equal surplus division (or, alternatively, individual rationality, regular aggregatte monotonicity and symmetry).

Finally, we explore the possibility to reconcile rationality, consistency and monotonicity with respect to the worth of the grand coalition. We provide a characterization of the family of single-valued solutions satisfying individual rationality and projected consistency together with regular or aggregate monotonicity. Despite these positive results, accommodate core selection and max consistency with monotonicity properties present some difficulties. It turns out that core selection, regular aggregate monotonicity and max consistency are incompatible on the domain of all games and also in the domain of balanced games. Moreover, if we restrict to convex games, although these three properties can be combined, core selection, equal surplus division and max consistency cannot.

The remainder of the paper is organized as follows. In Section 2, we introduce some solutions and review which of the aforementioned properties they satisfy. In Section 3, we analyze the (in)compatibility between rationality and consistency or monotonicity. In Section 4, we discuss how well combine rationality, consistency and monotonicity together. Some remarks and open questions conclude the paper.

\section{Solutions and properties}

The set of natural numbers $\mathbb{N}$ denotes the universe of potential players. A coalition is a non-empty finite subset of $\mathbb{N}$ and let $\mathcal{N}:=\{N|\emptyset \neq N \subseteq \mathbb{N}| N \mid,<\infty\}$ denote the set of all coalitions of $\mathbb{N}$. A transferable utility coalitional game (a game) is a pair $(N, v)$ where $N \in \mathcal{N}$ is the set of players and $v: 2^{N} \longrightarrow \mathbb{R}$ is the characteristic function that assigns to each coalition $S \subseteq N$ a real number $v(S)$, with the convention that $v(\emptyset)=0$. For simplicity of notation, and if no confusion arises, we write $v(i), v(i j), \ldots$ instead of $v(\{i\}), v(\{i, j\}), \ldots$ Given $N \in \mathcal{N}$ and $\emptyset \neq S \subseteq N$, the unanimity game $\left(N, u_{S}\right)$ associated to $S$ is defined as $u_{S}(R)=1$ if $S \subseteq R$ and $u_{S}(R)=0$ otherwise. Given a 
game $(N, v)$ and $N^{\prime} \subset N$, the subgame $\left(N^{\prime}, v_{\mid N^{\prime}}\right)$ is defined as $v_{\mid N^{\prime}}(S)=v(S)$ for all $S \subseteq N^{\prime}$. Given $S, T \in \mathcal{N}$, we use $S \subset T$ to indicate strict inclusion, that is, $S \subseteq T$ but $S \neq T$. By $|S|$ we denote the cardinality of the coalition $S \in \mathcal{N}$. For all $t \in \mathbb{R},|t|=t$ if $t \geq 0$ and $|t|=-t$ otherwise. By $\Gamma$ we denote the class of all games.

Given $N \in \mathcal{N}$, let $\mathbb{R}^{N}$ stands for the space of real-valued vectors indexed by $N$, $x=\left(x_{i}\right)_{i \in N}$, and for all $S \subseteq N, x(S)=\sum_{i \in S} x_{i}$, with the convention $x(\emptyset)=0$. Given $\emptyset \neq S \subseteq N, e_{S} \in \mathbb{R}^{N}$ is defined as $e_{S, i}=1$ if $i \in S$ and $e_{S, i}=0$ otherwise. For each $x \in \mathbb{R}^{N}$ and $T \subseteq N, x_{\mid T}$ denotes the restriction of $x$ to $T: x_{\mid T}=\left(x_{i}\right)_{i \in T} \in \mathbb{R}^{T}$. Given two vectors $x, y \in \mathbb{R}^{N}, x \geq y$ if $x_{i} \geq y_{i}$, for all $i \in N$.

The preimputation set of $(N, v)$ is defined by $X(N, v):=\left\{x \in \mathbb{R}^{N} \mid x(N)=v(N)\right\}$. A solution on a class of games $\Gamma^{\prime} \subseteq \Gamma$ is a correspondence $\sigma$ which associates with each game $(N, v) \in \Gamma^{\prime}$ a subset $\sigma(N, v)$ of $X(N, v)$. A solution $\sigma$ on $\Gamma^{\prime} \subseteq \Gamma$ is said to be single-valued if $|\sigma(N, v)|=1$ for all $(N, v) \in \Gamma^{\prime}$. The set of imputations is defined by $I(N, v):=\left\{x \in X(N, v) \mid x_{i} \geq v(\{i\})\right.$, for all $\left.i \in N\right\}$. A game with a non-empty imputation set is called essential. We denote by $\Gamma_{E}$ the class of essential games. The core (Gillies, 1953) of $(N, v)$ is the set of those imputations where each coalition gets at least its worth, that is $C(N, v):=\{x \in X(N, v) \mid x(S) \geq v(S)$ for all $S \subseteq N\}$. A game $(N, v)$ is balanced if it has a non-empty core. By $\Gamma_{B}$ we denote the class of balanced games. A game $(N, v)$ is convex if $v(S \cup T)+v(S \cap T) \geq v(S)+v(T)$, for all $S, T \subseteq N$. We denote by $\Gamma_{C}$ the class of convex games.

We introduce some well-known single-valued solutions defined on $\Gamma$. Let $N \in \mathcal{N}$ and $(N, v) \in \Gamma$. The Shapley value (Shapley, 1953), Sh, is defined by

$$
S h_{i}(N, v):=\sum_{S \subseteq N \backslash\{i\}} \frac{|S| !(|N|-|S|-1) !}{|N| !}(v(S \cup\{i\})-v(S)) \text { for all } i \in N .
$$

Let $N \in \mathcal{N}$ and $(N, v) \in \Gamma$. With any preimputation $x \in X(N, v)$ we associate the vector of excesses $e(S, x)=v(S)-x(S), \varnothing \neq S \subset N$, whose components are nonincreasingly ordered. The prenucleolus (Schmeidler, 1969), $\nu_{*}$, is the preimputation that lexicographically minimizes the vector of excesses over the set of preimputations. With any preimputation $x \in X(N, v)$ we associate the vector of per-capita excesses $\bar{e}(S, x)=\frac{v(S)-x(S)}{|S|}, \varnothing \neq S \subset N$, whose components are non-increasingly ordered. The per-capita prenucleolus (Grotte, 1970), $\bar{\nu}_{*}$, is the preimputation that lexicographically minimizes the vector of per-capita excesses over the set of preimputations. The equal division solution, $E D$, is defined by $E D_{i}(N, v)=\frac{v(N)}{|N|}$ for all $i \in N$. The center of imputations solution ${ }^{2}$ (Driessen and Funaki, 1991), CI, is defined by

$$
C I_{i}(N, v):=v(i)+\frac{1}{|N|}\left(v(N)-\sum_{i \in N} v(i)\right) \text { for all } i \in N .
$$

The weighted center of imputations solution, $C I^{w}$, relative to a list of positive

\footnotetext{
${ }^{2}$ This solution is also known as the equal surplus division rule (see, for instance, van den Brink, 2007, Béal et al., 2014 and Casajus and Huettner, 2014), or as the egalitarian value (see, for instance, Chun and Park, 2012).
} 
weights $w=\left(w_{i}\right)_{i \in \mathbb{N}} \in \mathbb{R}_{++}^{\mathbb{N}}$ is defined by

$$
C I_{i}^{w}(N, v):=v(i)+\frac{w_{i}}{\sum_{j \in N} w_{j}}\left(v(N)-\sum_{i \in N} v(i)\right) \text { for all } i \in N
$$

Note that when $w_{i}=1$ for all $i \in \mathbb{N}$, then $C I^{w}(N, v)=C I(N, v)$.

For our purposes, we introduce a single-valued solutions similar to the one provided by Calleja et al. (2012). Let $N \in \mathcal{N}$ and $(N, v) \in \Gamma$. The single-valued solution $\varphi$ is defined by

$$
\varphi(N, v):= \begin{cases}x_{I}^{v}+\frac{v(N)-\sum_{i \in N} v(i)}{v_{r}(N)-\sum_{i \in N} v(i)}\left(\nu_{*}\left(N, v_{r}\right)-x_{I}^{v}\right) & \text { if } v_{r}(N) \neq \sum_{i \in N} v(i), \\ C I(N, v) & \text { otherwise, }\end{cases}
$$

where $x_{I}^{v}=(v(1), v(2), \ldots, v(n)), N=\{1,2, \ldots, n\}$ and $\left(N, v_{r}\right)$ is the balanced game with smallest efficiency such that $v_{r}(S)=v(S)$ for all $S \subset N$.

Next, we introduce properties of solutions that apply to the games for a fixed population.

A single-valued solution $\sigma$ on $\Gamma^{\prime} \subseteq \Gamma$ satisfies

- Core selection (CS): if for all $N \in \mathcal{N}$ and all $(N, v) \in \Gamma^{\prime}$ with $C(N, v) \neq \emptyset$, it holds $\sigma(N, v) \in C(N, v)$.

- Individual rationality (IR): if for all $N \in \mathcal{N}$ and all $(N, v) \in \Gamma^{\prime}$ with $I(N, v) \neq$ $\emptyset$, it holds $\sigma(N, v) \in I(N, v)$.

Clearly, individual rationality does not imply core selection. Moreover, core selection does not imply individual rationality since a single-valued solution may select a non-individually rational allocation in a game with an empty core. Core selection and individual rationality are properties that concerns the rationality of a solution.

- Equal surplus division ${ }^{3}$ (ESD): if for all $N \in \mathcal{N}$ and all $(N, v),\left(N, v^{\prime}\right) \in \Gamma^{\prime}$ with $v(S)=v^{\prime}(S)$ for all $S \subset N$, it holds $\sigma\left(N, v^{\prime}\right)-\sigma(N, v)=\left(\frac{v^{\prime}(N)-v(N)}{|N|}, \ldots, \frac{v^{\prime}(N)-v(N)}{|N|}\right)$.

- Aggregate monotonicity (AM): if for all $N \in \mathcal{N}$ and all $(N, v),\left(N, v^{\prime}\right) \in \Gamma^{\prime}$ with $v(S)=v^{\prime}(S)$ for all $S \subset N$ and $v(N)<v^{\prime}(N)$, it holds that $\sigma(N, v) \leq \sigma\left(N, v^{\prime}\right)$.

Aggregate monotonicity (Megiddo, 1974) and equal surplus division are monotonicity properties with respect to the worth of the grand coalition. Aggregate monotonicity states that nobody's payoff strictly decrease when only the worth of the grand coalition grows, while equal surplus division imposes that agents share equally the raise of the worth of the grand coalition. Note that equal surplus division implies aggregate monotonicity. Most of single-valued solutions satisfying aggregate monotonicity, like $S h, \bar{\nu}_{*}$, $E D$ and $C I$, satisfy equal surplus division too (see Table 1).

\footnotetext{
${ }^{3}$ van den brink et al. (2012) call this property weak fairness. Recently, Arin and Katsev (2014) refer to it as strong aggregate monotonicity.
} 
Equal surplus division incorporates an idea of regularity. Whenever a set of players $N \in \mathcal{N}$ agree on how to distribute monotonically an amount $t \in \mathbb{R}$, representing the difference of efficiency between two games, they will respect this agreement independently of the games they finally face. According to equal surplus division this agreement consists of sharing $t$ equally among any set of players $N \in \mathcal{N}$. However, there are many other monotonic ways of distributing $t$.

Definition 1. A monotone path is the image of a function $f: \mathcal{N} \times \mathbb{R} \rightarrow \bigcup_{N \in \mathcal{N}} \mathbb{R}^{N}$ satisfying the following conditions: for all $N \in \mathcal{N}$ and all $t \in \mathbb{R}$,

(i) $f(N, 0)=(0, \ldots, 0) \in \mathbb{R}^{N}$,

(ii) $f(N, t) \in \mathbb{R}^{N}$ and $\sum_{i \in N} f_{i}(N, t)=t$,

(iii) if $t^{\prime} \in \mathbb{R}$ is such that $t^{\prime}>t$, then $f_{i}\left(N, t^{\prime}\right) \geq f_{i}(N, t)$ for all $i \in N$.

Note that a monotone path assigns non-negative (non-positive) vectors to positive (negative) real numbers.

Let $\mathcal{F}_{\text {mon }}$ denote the class of functions satisfying the above conditions. Examples of functions in $\mathcal{F}_{\text {mon }}$ widely used along the paper are:

1. For all $N \in \mathcal{N}$, all $t \in \mathbb{R}$ and all $i \in N$, define $\bar{f}_{i}(N, t)=\frac{t}{|N|}$.

$\bar{f}$ distributes $t$ equally among $N$.

2. Let $w \in \mathbb{R}_{++}^{\mathbb{N}}$ be a list of positive weights. For all $N \in \mathcal{N}$, all $t \in \mathbb{R}$ and all $i \in N$, define $f_{i}^{w}(N, t)=\frac{w_{i} \cdot t}{\sum_{j \in N} w_{j}}$.

$f^{w}$ distributes $t$ among $N$ proportionally according to their weights $w$.

3. Let $\pi$ be a permutation on $\mathbb{N}$. For all $N \in \mathcal{N}$ and all $t \in \mathbb{R}$, define $f^{\pi}(N, t)=t \cdot e_{\{j\}}$, being $j \in N$ such that $\pi(j) \geq \pi(i)$ for all $i \in N$.

$f^{\pi}$ assigns all the amount $t$ to the last player in $N$ according to $\pi$.

4. Let $\pi$ be a permutation on $\mathbb{N}$. For all $N \in \mathcal{N}$ and all $t \in \mathbb{R}$, define

$$
\widehat{f^{\pi}}(N, t)= \begin{cases}\left\lfloor\frac{t}{|N|}\right\rfloor \cdot e_{N}+\sum_{i \in S^{*}} e_{\{i\}}+(t \bmod |N|-\lfloor t \bmod |N|\rfloor) \cdot e_{\{k\}} & \text { if } t \geq 0 \\ -\widehat{f^{\pi}}(N,-t) & \text { if } t<0\end{cases}
$$

where $S^{*} \subset N$ is formed by the first $\lfloor t \bmod |N|\rfloor$ players according to $\pi$ (if there are) and $k \in N \backslash S^{*}$ with $\pi(k) \leq \pi(j)$, for all $j \in N \backslash S^{*}{ }^{4}$

The interpretation of $\widehat{f^{\pi}}$ when $t \geq 0$ is as follows: if $0 \leq t \leq 1$, then $\widehat{f^{\pi}}$ assigns the amount $t$ to the first player in $N$ according to $\pi$. If $1<t \leq 2$, the first player receives a unit of $t$ and the second player $t-1$, etc. If $t>|N|$, then after distributing one unit of $t$ to every player, it stars again given one additional unit to the first player, and so on until the amount $t$ is exhausted.

\footnotetext{
${ }^{4}$ For all $x, y \in \mathbb{R},\lfloor x\rfloor:=\max \{k \in \mathbb{Z} \mid k \leq x\}$, and $x \bmod y:=x-y \cdot\left\lfloor\frac{x}{y}\right\rfloor$.
} 
By using the notion of a monotone path, we introduce a new monotonicity property with respect to the worth of the grand coalition.

A single-valued solution $\sigma$ on $\Gamma^{\prime} \subseteq \Gamma$ satisfies

- Regular aggregate monotonicity (RAM): if there exists a monotone path $f \in \mathcal{F}_{\text {mon }}$ such that, for all $N \in \mathcal{N}$ and all $(N, v),\left(N, v^{\prime}\right) \in \Gamma^{\prime}$ with $v(S)=v^{\prime}(S)$ for all $S \subset N$, it holds $\sigma\left(N, v^{\prime}\right)-\sigma(N, v)=f\left(N, v^{\prime}(N)-v(N)\right)$.

Equal surplus division implies regular aggregate monotonicity with $f=\bar{f}$. Certainly, regular aggregate monotonicity implies aggregate monotonicity. Note that $C I^{w}$ satisfies regular aggregate monotonicity with $f=f^{w}$, but not equal surplus division. Moreover, $\varphi$ satisfies aggregate monotonicity but not regular aggregate monotonicity (see Table 1$).^{5}$

Next, we introduce consistency properties, that is, properties that relate the payoff vectors chosen for the games with variable population. Before doing this, we need to define the concept of a reduced game. We focus on three types of reduced games widely used to axiomatize solutions. The terminology is taken from Thomson (2003).

Let $N \in \mathcal{N},(N, v) \in \Gamma, x \in \mathbb{R}^{N}$ and $\emptyset \neq N^{\prime} \subset N$.

1. The max reduced game (Davis and Maschler, 1965) relative to $N^{\prime}$ at $x$ is the game $\left(N^{\prime}, r_{D M, x}^{N^{\prime}}(v)\right)$ defined by

$$
r_{D M, x}^{N^{\prime}}(v)(S):= \begin{cases}0 & \text { if } S=\emptyset, \\ \max _{Q \subseteq N \backslash N^{\prime}}\{v(S \cup Q)-x(Q)\} & \text { if } \emptyset \neq S \subset N^{\prime}, \\ v(N)-x\left(N \backslash N^{\prime}\right) & \text { if } S=N^{\prime} .\end{cases}
$$

2. The complement reduced game (Moulin, 1985) relative to $N^{\prime}$ at $x$ is the game $\left(N^{\prime}, r_{M, x}^{N^{\prime}}(v)\right)$ defined by

$$
r_{M, x}^{N^{\prime}}(v)(S):= \begin{cases}0 & \text { if } S=\emptyset, \\ v\left(S \cup N \backslash N^{\prime}\right)-x\left(N \backslash N^{\prime}\right) & \text { if } \emptyset \neq S \subseteq N^{\prime} .\end{cases}
$$

3. The projected reduced game (Funaki, 1998) relative to $N^{\prime}$ at $x$ is the game $\left(N^{\prime}, r_{F, x}^{N^{\prime}}(v)\right)$ defined by

$$
r_{F, x}^{N^{\prime}}(v)(S):= \begin{cases}v(S) & \text { if } S \subset N^{\prime} \\ v(N)-x\left(N \backslash N^{\prime}\right) & \text { if } S=N^{\prime}\end{cases}
$$

In the max-reduced game (relative to $N^{\prime}$ at $x$ ), the worth of a coalition $S \subset N^{\prime}$ is determined under the assumption that $S$ can choose the best partners in $N \backslash N^{\prime}$, provided that it pays them their components of $x$. The complement and the projected reduced games represent the two extreme cases. In the complement reduced game each coalition $S \subset N^{\prime}$ is required to join all the members of $N \backslash N^{\prime}$ while, by contrast, in the projected reduced game when players in $N \backslash N^{\prime}$ leave the game, no cooperation with them is possible anymore.

A single-valued solution $\sigma$ on $\Gamma^{\prime} \subseteq \Gamma$ satisfies

\footnotetext{
${ }^{5}$ This is because $\varphi$ satisfies individual rationality and core selection and these two properties are incompatible with regular aggregate monotonicity (see Theorem 3).
} 
- Max consistency (M-CON): if for all $N \in \mathcal{N}$, all $(N, v) \in \Gamma^{\prime}$, all $\emptyset \neq N^{\prime} \subset N$, and $x=\sigma(N, v)$, then $\left(N^{\prime}, r_{D M, x}^{N^{\prime}}(v)\right) \in \Gamma^{\prime}$ and $x_{\mid N^{\prime}}=\sigma\left(N^{\prime}, r_{D M, x}^{N^{\prime}}(v)\right)$.

- Complement consistency (C-CON): if for all $N \in \mathcal{N}$, all $(N, v) \in \Gamma^{\prime}$, all $\emptyset \neq N^{\prime} \subset N$, and $x=\sigma(N, v)$, then $\left(N^{\prime}, r_{M, x}^{N^{\prime}}(v)\right) \in \Gamma^{\prime}$ and $x_{\mid N^{\prime}}=\sigma\left(N^{\prime}, r_{M, x}^{N^{\prime}}(v)\right)$.

- Projected consistency (P-CON): if for all $N \in \mathcal{N}$, all $(N, v) \in \Gamma^{\prime}$, all $\emptyset \neq N^{\prime} \subset$ $N$, and $x=\sigma(N, v)$, then $\left(N^{\prime}, r_{F, x}^{N^{\prime}}(v)\right) \in \Gamma^{\prime}$ and $x_{\mid N^{\prime}}=\sigma\left(N^{\prime}, r_{F, x}^{N^{\prime}}(v)\right)$.

Table 1 below summarizes whether or not the single-valued solutions already introduced satisfy the properties mentioned so far. Some cells of the table are fulfilled by means of results provided in the next sections of this work.

Table 1. Properties of solutions defined on $\Gamma$

\begin{tabular}{lccccccc}
\hline & $S h$ & $\nu_{*}$ & $\bar{\nu}_{*}$ & $E D$ & $C I$ & $C I^{w}$ & $\varphi$ \\
\hline CS & $\times$ & $\checkmark$ & $\checkmark$ & $\times$ & $\times$ & $\times$ & $\checkmark$ \\
IR & $\times$ & $\times$ & $\times$ & $\times$ & $\checkmark$ & $\checkmark$ & $\checkmark$ \\
ESD & $\checkmark$ & $\times$ & $\checkmark$ & $\checkmark$ & $\checkmark$ & $\times$ & $\times($ Th. 3) \\
RAM & $\checkmark$ & $\times$ & $\checkmark$ & $\checkmark$ & $\checkmark$ & $\checkmark$ & $\times($ Th. 3) \\
AM & $\checkmark$ & $\times$ & $\checkmark$ & $\checkmark$ & $\checkmark$ & $\checkmark$ & $\checkmark$ \\
M-CON & $\times$ & $\checkmark$ & $\times($ Th. 8$)$ & $\checkmark$ & $\times$ & $\times$ & $\times($ Cor. 1$)$ \\
C-CON & $\times$ & $\times($ Th. 4$)$ & $\times($ Th. 4$)$ & $\checkmark$ & $\times$ & $\times$ & $\times($ Cor. 1$)$ \\
P-CON & $\times$ & $\times($ Th. 5) & $\times($ Th. 5) & $\checkmark$ & $\checkmark($ Th. 6) & $\checkmark($ Th. 6) & $\times($ Cor. 1$)$ \\
\hline
\end{tabular}

\section{Rationality and monotonicity or consistency}

From the observation of Table 1, it turns out that combining monotonicity with consistency properties is always possible since ED satisfies equal surplus division (and, therefore, regular aggregate monotonicity and aggregate monotonicity), max consistency, complement consistency and projected consistency.

If we focus on how well properties of rationality combine with properties of monotonicity, note that core selection and equal surplus division are compatible by means of $\bar{\nu}_{*}$, and individual rationality and equal surplus division by means of $C I$. In fact, individual rationality and equal surplus division characterize $C I$.

Theorem 1. The center of imputations solution is the unique single-valued solution on $\Gamma$ that satisfies individual rationality and equal surplus division.

Proof. It is easy to check that $C I$ satisfies individual rationality and equal surplus division. Let $\sigma$ be a single-valued solution on $\Gamma$ satisfying these two properties, $N \in \mathcal{N}$ and $(N, v)$ be a game. If $v(N)=\sum_{i \in N} v(i)$ then, by individual rationality, $\sigma_{i}(N, v)=$ $v(i)=C I_{i}(N, v)$ for all $i \in N$. On the other hand, if $v(N) \neq \sum_{i \in N} v(i)$, define the game $\left(N, v^{\prime}\right)$ as $v^{\prime}(S)=v(S)$ for all $S \neq N$, and $v^{\prime}(N)=\sum_{i \in N} v(i)$. For all $i \in N$, applying 
individual rationality we have $\sigma_{i}\left(N, v^{\prime}\right)=v(i)$ and, by equal surplus division, we obtain

$$
\begin{aligned}
\sigma_{i}(N, v) & =\sigma_{i}\left(N, v^{\prime}\right)+\frac{1}{|N|}\left(v(N)-v^{\prime}(N)\right) \\
& =v(i)+\frac{1}{|N|}\left(v(N)-\sum_{i \in N} v(i)\right) \\
& =C I_{i}(N, v) .
\end{aligned}
$$

Clearly, individual rationality and equal surplus division are independent on $\Gamma$ (see Table 1). CI can also be characterized by using regular aggregate monotonicity together with individual rationality and symmetry.

A single-valued solution $\sigma$ on $\Gamma^{\prime} \subseteq \Gamma$ satisfies

- Symmetry (SYM) if for all $N \in \mathcal{N}$, all $(N, v) \in \Gamma^{\prime}$ and all $i, j \in N$, if $v(S \cup i)=$ $v(S \cup j)$ for all $S \subseteq N \backslash\{i, j\}$, then $\sigma_{i}(N, v)=\sigma_{j}(N, v)$.

Next we show that regular aggregate monotonicity and symmetry imply equal surplus division.

Proposition 1. On the domain of all games $\Gamma$, regular aggregate monotonicity together with symmetry imply equal surplus division.

Proof. Let $\sigma$ be a single-valued solution on $\Gamma$ satisfying regular aggregate monotonicity and symmetry. Let $N \in \mathcal{N}, t \in \mathbb{R}$ and $(N, v)$ be a game such that $v(S)=|S|$ for all $S \subseteq N$. By symmetry, $\sigma(N, v)=\left(\frac{v(N)}{|N|}, \ldots, \frac{v(N)}{|N|}\right)$. Now, consider the game $\left(N, v^{\prime}\right)$ defined by $v^{\prime}=v+t \cdot u_{N}$. Since all players are also symmetric in $\left(N, v^{\prime}\right)$, by symmetry it holds

$$
\sigma\left(N, v^{\prime}\right)=\left(\frac{v(N)+t}{|N|}, \ldots, \frac{v(N)+t}{|N|}\right)=\sigma(N, v)+\left(\frac{t}{|N|}, \ldots, \frac{t}{|N|}\right) .
$$

By regular aggregate monotonicity, there exists $f \in \mathcal{F}_{\text {mon }}$ such that

$$
\sigma\left(N, v^{\prime}\right)=\sigma(N, v)+f(N, t) .
$$

From (1) and (2) it follows that $f(N, t)=\left(\frac{t}{|N|}, \ldots, \frac{t}{|N|}\right)$, which means that $\sigma$ satisfies equal surplus division.

Theorem 1 and Proposition 1, together with the fact that $C I$ satisfies symmetry, lead to the next characterization. ${ }^{6}$

Theorem 2. The center of imputations solution is the unique single-valued solution on $\Gamma$ that satisfies individual rationality, regular aggregate monotonicity and symmetry.

\footnotetext{
${ }^{6}$ As we have commented in the introduction, recent axiomatic characterizations of $C I$ can be found in Béal et al. (2014), Casajus and Huettner (2014), Chun and Park (2012), and van den Brink (2007).
} 
The properties in Theorem 2 are independent. ${ }^{7}$

Theorem 1, together with the fact that $C I$ does not satisfy core selection, imply that core selection, individual rationality and equal surplus division are incompatible. However, core selection, individual rationality and aggregate monotonicity are compatible by means of $\varphi$. The next result shows that core selection and individual rationality are incompatible with regular aggregate monotonicity.

Theorem 3. For $|N| \geq 3$ there is no single-valued solution on $\Gamma$ that satisfies core selection, individual rationality and regular aggregate monotonicity.

Proof. Suppose, on the contrary, that there exists a single-valued solution $\sigma$ satisfying core selection, individual rationality and regular aggregate monotonicity on $\Gamma$.

Let $\left(N, v^{1}\right)$ be a game with set of players $N=\{1,2,3\}$ and characteristic function: $v^{1}(12)=v^{1}(13)=v^{1}(123)=1$ and $v^{1}(S)=0$ otherwise. Clearly, $C\left(N, v^{1}\right)=$ $\{(1,0,0)\}$ and, by core selection, $\sigma\left(N, v^{1}\right)=(1,0,0)$. Now, define the game $\left(N,\left(v^{1}\right)^{I}\right)$ as $\left(v^{1}\right)^{I}=v^{1}+(-1) \cdot u_{N}$. Since $I\left(N,\left(v^{1}\right)^{I}\right)=\{(0,0,0)\}$, by individual rationality, $\sigma\left(N,\left(v^{1}\right)^{I}\right)=(0,0,0)$. By regular aggregate monotonicity, there exists $f \in \mathcal{F}_{\text {mon }}$ such that $\sigma\left(N,\left(v^{1}\right)^{I}\right)-\sigma\left(N, v^{1}\right)=f(N,-1)$, from which it follows $f(N,-1)=(-1,0,0)$.

Let us now consider the game $\left(N, v^{2}\right)$ with set of players $N=\{1,2,3\}$ and characteristic function $v^{2}(12)=v^{2}(23)=v^{2}(123)=1$ and $v^{2}(S)=0$ otherwise. Let $\left(N,\left(v^{2}\right)^{I}\right)$ be defined as $\left(v^{2}\right)^{I}=v^{2}+(-1) \cdot u_{N}$. Following similar arguments than before we have, by core selection, $\sigma\left(N, v^{2}\right)=(0,1,0)$, by individual rationality, $\sigma\left(N,\left(v^{2}\right)^{I}\right)=(0,0,0)$ and by regular aggregate monotonicity, $f(N,-1)=(0,-1,0)$, in contradiction with $f(N,-1)=(-1,0,0)$.

Table 2 summarizes the (in)compatibilities of properties of rationality combined with properties of monotonicity for single-valued solutions on $\Gamma$.

Table 2. (In)compatibilities of rationality and monotonicity on $\Gamma$

\begin{tabular}{l|ccc} 
& CS & IR & CS + IR \\
\hline ESD & $\bar{\nu}_{*}$ & $C I$ & $\times($ Th. 1) \\
RAM & $\bar{\nu}_{*}$ & $C I$ & $\times($ Th. 3) \\
AM & $\bar{\nu}_{*}$ & $C I$ & $\varphi$
\end{tabular}

Finally, we study the compatibility for single-valued solutions of properties of rationality together with one of the three versions of consistency we have introduced. Although formally it would be admissible to consider two consistency properties simultaneously, from a behavioral point of view it has no sense that players agree in two different ways of reducing the game.

\footnotetext{
${ }^{7}$ ED satisfies regular aggregate monotonicity and symmetry but not individual rationality, $\varphi$ satisfies individual rationality and symmetry but not regular aggregate monotonicity and, for a suitable list of positive weights $w, C I^{w}$ satisfies individual rationality and regular aggregate monotonicity but not symmetry.
} 
On the domain of all games, $\nu_{*}$ satisfies core selection and max consistency (Potters, 1991). Moreover, the core has been characterized using max consistency (Peleg, 1986), complement consistency (Tadenuma, 1992) and projected consistency (Llerena and Rafels, 2007). But, surprisingly, for single-valued solutions core selection together with either complement consistency or projected consistency are incompatible.

Theorem 4. For $|N| \geq 3$ there is no single-valued solution on $\Gamma$ that satisfies core selection and complement consistency.

Proof. Suppose, on the contraty, that there exists a single-valued solution $\sigma$ satisfying core selection and complement consistency on $\Gamma$. Let $\left(N, v^{1}\right)$ and $\left(N, v^{2}\right)$ be two balanced games with set of players $N=\{1,2,3\}$ and characteristic functions as follows: $v^{1}(1)=$ $v^{2}(2)=0, v^{1}(2)=v^{2}(1)=1$ and $v^{1}(S)=v^{2}(S)$ for any other $S \subseteq N$, being $v^{1}(3)=$ $1, v^{1}(12)=0, v^{1}(13)=v^{1}(23)=1$ and $v^{1}(N)=2$. Notice that $C\left(N, v^{1}\right)=\{(0,1,1)\}$ and $C\left(N, v^{2}\right)=\{(1,0,1)\}$. By core selection, $\sigma\left(N, v^{1}\right)=(0,1,1)$ and $\sigma\left(N, v^{2}\right)=(1,0,1)$. Let $N^{\prime}=\{1,2\}$. By complement consistency,

$$
\sigma\left(N^{\prime}, r_{M,(0,1,1)}^{N^{\prime}}\left(v^{1}\right)\right)=(0,1) \in C\left(N^{\prime}, r_{M,(0,1,1)}^{N^{\prime}}\left(v^{1}\right)\right)
$$

and

$$
\sigma\left(N^{\prime}, r_{M,(1,0,1)}^{N^{\prime}}\left(v^{2}\right)\right)=(1,0) \in C\left(N^{\prime}, r_{M,(1,0,1)}^{N^{\prime}}\left(v^{2}\right)\right)
$$

$\operatorname{But}\left(N^{\prime}, r_{M,(0,1,1)}^{N^{\prime}}\left(v^{1}\right)\right)=\left(N^{\prime}, r_{M,(1,0,1)}^{N^{\prime}}\left(v^{2}\right)\right)$, which leads a contradiction.

Theorem 5. For $|N| \geq 3$ there is no single-valued solution on $\Gamma$ that satisfies core selection and projected consistency.

Proof. Suppose, on the contrary, that there exits a single-valued solutions $\sigma$ satisfying core selection and projected consistency on $\Gamma$. Let $\left(N, v^{1}\right)$ and $\left(N, v^{2}\right)$ be two balanced games with set of players $N=\{1,2,3\}$ and characteristic functions as follows: $v^{1}(i)=0$ for all $i \in N, v^{1}(12)=v^{1}(13)=v^{1}(123)=1$ and $v^{1}(23)=0$, and $v^{2}(S)=v^{1}(S)$, for $S \neq\{13\}$ and $S \neq\{23\}, v^{2}(13)=0$ and $v^{2}(23)=1$. Notice that $C\left(N, v^{1}\right)=\{(1,0,0)\}$ and $C\left(N, v^{2}\right)=\{(0,1,0)\}$. By core selection, $\sigma\left(N, v^{1}\right)=(1,0,0)$ and $\sigma\left(N, v^{2}\right)=(0,1,0)$. Let $N^{\prime}=\{1,2\}$. By projected consistency,

$$
\sigma\left(N^{\prime}, r_{F,(1,0,0)}^{N^{\prime}}\left(v^{1}\right)\right)=(1,0) \in C\left(N^{\prime}, r_{F,(1,0,0)}^{N^{\prime}}\left(v^{1}\right)\right)
$$

and

$$
\sigma\left(N^{\prime}, r_{F,(0,1,0)}^{N^{\prime}}\left(v^{2}\right)\right)=(0,1) \in C\left(N^{\prime}, r_{F,(0,1,0)}^{N^{\prime}}\left(v^{2}\right)\right) .
$$

$\operatorname{But}\left(N^{\prime}, r_{F,(1,0,0)}^{N^{\prime}}\left(v^{1}\right)\right)=\left(N^{\prime}, r_{F,(0,1,0)}^{N^{\prime}}\left(v^{2}\right)\right)$, which leads a contradiction.

If we replace core selection by individual rationality, Hwang (2013) shows that there is no single-valued solution satisfying simultaneously individual rationality and max consistency, or individual rationality and complement consistency on $\Gamma$. However, individual rationality and projected consistency are compatible by means of $C I$ (see Theorem 6). Thus, combining Theorem 5 and Hwang's results we obtain the following corollary. 
Corollary 1. For $|N| \geq 3$ there is no single-valued solution on $\Gamma$ that satisfies core selection, individual rationality and either max consistency or complement consistency or projected consistency.

Table 3 summarizes the (in)compatibilities of properties of rationality combined with properties of consistency for single-valued solutions on $\Gamma$.

Table 3. (In)compatibilities of rationality and consistency on $\Gamma$

\begin{tabular}{l|ccc} 
& CS & IR & CS + IR \\
\hline M-CON & $\nu_{*}$ & $\times$ & $\times($ Cor. 1$)$ \\
C-CON & $\times($ Th. 4) & $\times$ & $\times($ Cor. 1$)$ \\
P-CON & $\times($ Th. 5) & $C I($ Th. 6) & $\times($ Cor. 1$)$
\end{tabular}

\section{Rationality, monotonicity and consistency}

The main issue of this section is to study if it is possible to combine for a single-valued solution a property of rationality with a property of monotonicity and a property of consistency.

Table 3 above shows that core selection can only be combined with max consistency, and individual rationality with projected consistency. First, we focus on the compatibility of individual rationality, projected consistency and monotonicity properties with respect to the worth of the grand coalition.

\subsection{Possibility results}

We begin characterizing the family of single-valued solutions satisfying individual rationality, regular aggregate monotonicity and projected consistency introducing a generalization of $C I$.

Definition 2. Let $f \in \mathcal{F}_{\text {mon }}$. The $f$-center of imputations, $C I^{f}$, is defined as follows: for all $N \in \mathcal{N}$, all $(N, v) \in \Gamma$ and all $i \in N$,

$$
C I_{i}^{f}(N, v):=v(i)+f_{i}\left(N, v(N)-\sum_{i \in N} v(i)\right) .
$$

$C I^{f}$ can be interpreted as a two stage rule: after assigning to every player what they can achieve for themselves alone, it distributes monotonically (but not necessarily equally) what is left of the gains of cooperation. Note that if $f=\bar{f}$ or $f=f^{w}$ we recover $C I$ or $C I^{w}$, respectively.

In order to select the subset of $C I^{f}$ solutions that are projected consistent we introduce the notion of consistent monotone path. ${ }^{8}$

\footnotetext{
${ }^{8}$ Not all $C I^{f}$ solutions are projected consistent. Let us show an example. Take a permutation $\pi$ on $\mathbb{N}$. For all $N \in \mathcal{N}$ and all $t \in \mathbb{R}$, define $f^{\sharp}(N, t)=t \cdot e_{\{j\}}$, being $j \in N$ such that $\pi(j) \leq \pi(i)$ for all $i \in N$ if $|N|$ is even, and $\pi(j) \geq \pi(i)$ for all $i \in N$ if $|N|$ is odd.

If the cardinality of $\bar{N}$ is even, $f^{\sharp}$ assigns all the amount $t$ to the first player in $N$ according to $\pi$;
} 
Definition 3. A monotone path $f \in \mathcal{F}_{\text {mon }}$ is said to be consistent if for all $N \in \mathcal{N}$, all $N^{\prime} \subset N$ and all $t \in \mathbb{R}$, it holds

$$
f_{j}\left(N^{\prime}, \sum_{i \in N^{\prime}} f_{i}(N, t)\right)=f_{j}(N, t) \text { for all } j \in N^{\prime} .
$$

Condition (3) has been used by Hokari (2002) to provide non-symmetric and nonhomogeneous generalizations of the egalitarian solution of Dutta and Ray (1989) on the domain of convex games. It is not difficult to check that $\bar{f}, f^{w}$ and $\widehat{f^{\pi}}$ are consistent monotone paths, while $f^{\sharp}$ is not (see footnote 8).

For our characterization result we need introduce the notion of additive path.

Definition 4. A monotone path $f \in \mathcal{F}_{\text {mon }}$ is said to be additive if for all $N \in \mathcal{N}$ and all $t, t^{\prime} \in \mathbb{R}$, it holds $f\left(N, t+t^{\prime}\right)=f(N, t)+f\left(N, t^{\prime}\right)$.

Examples of additive monotone paths are $\bar{f}, f^{w}$, and $f^{\sharp}$, while $\widehat{f^{\pi}}$ is not. ${ }^{9}$

Theorem 6. A single-valued solution $\sigma$ satisfies individual rationality, regular aggregate monotonicity and projected consistency on $\Gamma$ if and only if there exists an additive and consistent monotone path $f \in \mathcal{F}_{\text {mon }}$ such that $\sigma=C I^{f}$.

Proof. Let $\sigma$ be a single-valued solution satisfying individual rationality, regular aggregate monotonicity and projected consistent on $\Gamma$.

Let $N \in \mathcal{N}$ and $(N, v) \in \Gamma$. Define $\left(N, v^{\prime}\right)$ as $v^{\prime}(S)=v(S)$ for all $S \subset N$, and $v^{\prime}(N)=\sum_{i \in N} v(i)$. By individual rationality, $\sigma_{i}\left(N, v^{\prime}\right)=v(i)$ for all $i \in N$. By regular aggregate monotonicity, there exists a monotone path $f \in \mathcal{F}_{\text {mon }}$ such that, for all $i \in N$,

$$
\sigma_{i}(N, v)=\sigma_{i}\left(N, v^{\prime}\right)+f_{i}\left(N, v(N)-v^{\prime}(N)\right)=v(i)+f_{i}\left(N, v(N)-\sum_{i \in N} v(i)\right) .
$$

Let us see that $f$ is additive. Let $N \in \mathcal{N}$ and $t, t^{\prime} \in \mathbb{R}$. Consider three games $(N, v),\left(N, v^{\prime}\right)$ and $\left(N, v^{\prime \prime}\right)$ defined as follows: for all $S \subset N, v(S)=v^{\prime}(S)=v^{\prime \prime}(S)$, $v(N)-v^{\prime}(N)=t$ and $v^{\prime}(N)-v^{\prime \prime}(N)=t^{\prime}$. Then,

$$
\begin{aligned}
f\left(N, t+t^{\prime}\right) & =f\left(N,\left(v^{\prime}(N)+t\right)-\left(v^{\prime}(N)-t^{\prime}\right)\right) \\
& =f\left(N, v(N)-v^{\prime \prime}(N)\right) \\
& =\sigma(N, v)-\sigma\left(N, v^{\prime \prime}\right) \\
& =\sigma(N, v)-\sigma\left(N, v^{\prime}\right)+\sigma\left(N, v^{\prime}\right)-\sigma\left(N, v^{\prime \prime}\right) \\
& =f\left(N, v(N)-v^{\prime}(N)\right)+f\left(N, v^{\prime}(N)-v^{\prime \prime}(N)\right) \\
& =f(N, t)+f\left(N, t^{\prime}\right),
\end{aligned}
$$

otherwise, $f^{\sharp}$ assigns $t$ to the last player in $N$ according to $\pi$. Then, $f^{\sharp} \in \mathcal{F}_{\text {mon }}$.

Consider the single-valued solution $C I^{f^{\sharp}}$ and the game $(N, v)$, with $N=\{1,2,3\}, v(i)=0$ for all $i \in N, v(12)=v(13)=v(123)=1$ and $v(23)=0$. Since $|N|$ is odd, $C I^{f^{\sharp}}(N, v)=(0,0,1)$. Now, take the projected reduced game $\left(N^{\prime}, r_{F, x}^{N^{\prime}}(v)\right)$, being $N^{\prime}=\{2,3\}$ and $x=(0,0,1)$. Since $\left|N^{\prime}\right|$ is even, $\sigma\left(N^{\prime}, r_{F, x}^{N^{\prime}}(v)\right)=(1,0) \neq(0,1)$, contradicting projected consistency.

${ }^{9}$ Note that $\widehat{f^{\pi}}$ is consistent but not additive, and $f^{\sharp}$ is additive but not consistent.

Let us introduced a monotone path that is neither additive nor consistent. For all $N \in \mathcal{N}$ and all $t \in \mathbb{R}$, define $f^{*}(N, t):=\widehat{f^{\pi}}(N, t)$ if $|N| \leq 3$, and $f^{*}(N, t):=\bar{f}(N, t)$ otherwise. According to $f^{*}$, different sets of players reach different agreements on how to distribute $t \in \mathbb{R}$. 
where third and fifth equalities follow from $\sigma$ satisfying regular aggregate monotonicity.

To check that $f$ is consistent, let $N \in \mathcal{N}, N^{\prime} \subset N$ and $t \in \mathbb{R}$. Consider a game $(N, v)$ such that $v(N)-\sum_{i \in N} v(i)=t$. Let us denote $x=\sigma(N, v)$. For all $j \in N^{\prime}$, from (4) we have

$$
\sigma_{j}(N, v)=v(j)+f_{j}\left(N, v(N)-\sum_{i \in N} v(i)\right)=v(j)+f_{j}(N, t)
$$

and

$$
\sigma_{j}\left(N^{\prime}, r_{F, x}^{N^{\prime}}(v)\right)=r_{F, x}^{N^{\prime}}(v)(j)+f_{j}\left(N^{\prime}, r_{F, x}^{N^{\prime}}(v)\left(N^{\prime}\right)-\sum_{i \in N^{\prime}} r_{F, x}^{N^{\prime}}(v)(i)\right)
$$

From the definition of the projected reduced game, the efficiency of $\sigma$ and (4), we have

$$
\begin{aligned}
r_{F, x}^{N^{\prime}}(v)\left(N^{\prime}\right)-\sum_{i \in N^{\prime}} r_{F, x}^{N^{\prime}}(v)(i) & =\sum_{i \in N^{\prime}} \sigma_{i}(N, v)-\sum_{i \in N^{\prime}} v(i) \\
& =\sum_{i \in N^{\prime}}\left[v(i)+f_{i}\left(N, v(N)-\sum_{i \in N} v(i)\right)\right]-\sum_{i \in N^{\prime}} v(i) \\
& =\sum_{i \in N^{\prime}} f_{i}\left(N, v(N)-\sum_{i \in N} v(i)\right)
\end{aligned}
$$

and expression (6) becomes

$$
\sigma_{j}\left(N^{\prime}, r_{F, x}^{N^{\prime}}(v)\right)=v(j)+f_{j}\left(N^{\prime}, \sum_{i \in N^{\prime}} f_{i}(N, t)\right) .
$$

By projected consistency, expressions (5) and (8) must coincide and thus

$$
f_{j}(N, t)=f_{j}\left(N^{\prime}, \sum_{i \in N^{\prime}} f_{i}(N, t)\right)
$$

which proves that $f$ is consistent.

Hence, $\sigma=C I^{f}$ being $f \in \mathcal{F}_{\text {mon }}$ an additive and consistent monotone path.

To show the reverse implication, let $\sigma$ be a single-valued solution on $\Gamma$ such that $\sigma=C I^{f}$, for some additive and consistent monotone path $f \in \mathcal{F}_{\text {mon }}$.

Let $(N, v)$ be an essential game. Then, $v(N)-\sum_{i \in N} v(i) \geq 0$ and thus, from the monotonicity of $f, f_{i}\left(N, v(N)-\sum_{i \in N} v(i)\right) \geq 0$, for all $i \in N$. Hence, $\sigma_{i}(N, v)=$ $v(i)+f_{i}\left(N, v(N)-\sum_{i \in N} v(i)\right) \geq v(i)$ for all $i \in N$, which proves individual rationality.

To see regular aggregate monotonicity consider two games $(N, v),\left(N, v^{\prime}\right)$ such that $v(S)=v^{\prime}(S)$, for all $S \subset N$. Taking into account the additivity of $f$ we have, for all $i \in N$,

$$
\begin{aligned}
\sigma_{i}(N, v)-\sigma_{i}\left(N, v^{\prime}\right) & =f_{i}\left(N, v(N)-\sum_{i \in N} v(i)\right)-f_{i}\left(N, v^{\prime}(N)-\sum_{i \in N} v(i)\right) \\
& =f_{i}\left(N, v(N)-\sum_{i \in N} v(i)\right)+f_{i}\left(N,-v^{\prime}(N)+\sum_{i \in N} v(i)\right) \\
& =f_{i}\left(N, v(N)-v^{\prime}(N)\right) .
\end{aligned}
$$


This, together with $f \in \mathcal{F}_{\text {mon }}$, proves regular aggregate monotonicity.

To check projected consistency, let $(N, v)$ be a game and $\emptyset \neq N^{\prime} \subset N$. Let us denote $x=\sigma(N, v)$. For all $j \in N^{\prime}$ we have

$$
\begin{aligned}
\sigma_{j}\left(N^{\prime}, r_{F, x}^{N^{\prime}}(v)\right) & =r_{F, x}^{N^{\prime}}(v)(j)+f_{j}\left(N^{\prime}, r_{F, x}^{N^{\prime}}(v)\left(N^{\prime}\right)-\sum_{i \in N^{\prime}} r_{F, x}^{N^{\prime}}(v)(i)\right) \\
& =v(j)+f_{j}\left(N^{\prime}, \sum_{i \in N^{\prime}} f_{i}\left(N, v(N)-\sum_{i \in N} v(i)\right)\right) \\
& =v(j)+f_{j}\left(N, v(N)-\sum_{i \in N} v(i)\right) \\
& =\sigma_{j}(N, v),
\end{aligned}
$$

where the second equality comes from (7) and the last before from the consistency of $f$. This proves projected consistency.

The properties in Theorem 6 are independent. ${ }^{10}$

Instances of single-valued solutions satisfying individual rationality, regular aggregate monotonicity and projected consistency are $C I$ and $C I^{w}$. Hence, since $C I$ is the unique single-valued solution that obeys individual rationality and equal surplus division (Theorem 1), individual rationality and equal surplus division imply projected consistency.

The next corollary, which comes straightforwardly from the proof of Theorem 6, extends the characterization in Theorem 1 when we replace equal surplus division by regular aggregate monotonicity.

Corollary 2. A single-valued solution $\sigma$ satisfies individual rationality and regular aggregate monotonicity on $\Gamma$ if and only if there exists an additive monotone path $f \in \mathcal{F}_{\text {mon }}$ such that $\sigma=C I^{f}$.

It is worth to mention that $C I^{\widehat{f^{\pi}}}$ satisfies individual rationality, aggregate monotonicity and projected consistency but not regular aggregate monotonicity. Indeed, there are other single-valued solutions which do not belong to the family of $f$ - center of imputations solutions that also satisfy individual rationality, aggregate monotonicity and projected consistency. ${ }^{11}$ Thus, there is a room to look for a characterization of the

${ }^{10} C I^{f^{\sharp}}$ satisfies individual rationality and regular aggregate monotonicity but not projected consistency, $C I^{f^{\pi}}$ satisfies individual rationality and projected consistency but not regular aggregate monotonicity (since $\widehat{f^{\pi}}$ is not additive), and ED satisfies regular aggregate monotonicity and projected consistency but not individual rationality.

${ }^{11}$ Define the single-valued solution $\rho$ as follows. For all $N \in \mathcal{N}$, all $(N, v) \in \Gamma$ and all $i \in N$

$$
\rho_{i}(N, v):= \begin{cases}v(i)+\left(v(N)-\sum_{i \in N} v(i)\right) & \text { if } i=i^{*} \\ v(i) & \text { otherwise }\end{cases}
$$

where $i^{*} \in N$ is such that $v\left(i^{*}\right) \geq v(i)$, for all $i \in N$, and $i^{*} \leq j$, for all $j \in N$ with $v\left(i^{*}\right)=v(j)$.

It is easy to verify that $\rho$ satisfies individual rationality, aggregate monotonicity and projected consistency. However, there is no $f \in \mathcal{F}_{m o n}$ such that $\rho=C I^{f}$. To check this, consider the games $\left(N, v^{1}\right),\left(N, v^{2}\right)$ with player set $N=\{1,2\}$ and characteristic functions: $v^{1}(1)=v^{2}(2)=1$, $v^{1}(2)=v^{2}(1)=0$ and $v^{1}(N)=v^{2}(N)=2$. Clearly, (a) $\rho_{1}\left(N, v^{1}\right)=v^{1}(1)+1$ and (b) $\rho_{1}\left(N, v^{2}\right)=v^{2}(1)$. Suppose, on the contrary, that there exists $f \in \mathcal{F}_{\text {mon }}$ such that $\rho=C I^{f}$. Then, from $(\mathrm{a}), f_{1}(N, 1)=1$ and, from (b), $f_{1}(N, 1)=0$, getting a contradiction. 
class of single-valued solutions satisfying these three properties. To this aim, let us first introduce some definitions.

Given $(N, v) \in \Gamma$, we define the associated game $\left(N, v^{I}\right)$ as follows: $v^{I}(S)=v(S)$ for all $S \subset N$, and $v^{I}(N)=\sum_{i \in N} v(i)$. Notice that $(N, v)$ can be written as $v=$ $v^{I}+\left(v(N)-v^{I}(N)\right) \cdot u_{N}$. Denote $\Gamma_{I}:=\left\{(N, v) \in \Gamma \mid v(N)=\sum_{i \in N} v(i)\right\}$.

Definition 5. A monotone $\Gamma_{I}$ - selection is a function $F: \Gamma_{I} \rightarrow \mathcal{F}_{\text {mon }}$ that associates with each game $(N, v) \in \Gamma_{I}$ a monotone path $F(N, v) \in \mathcal{F}_{\text {mon }}$.

By simplicity, and if there is no confusion, we denote $F(N, v)$ by $f^{v}$. Notice that different games in the set $\Gamma_{I}$ may have associated different monotone paths.

Making use of the notion of a monotone $\Gamma_{I}$ - selection, we introduce the $F$ - center of imputations solution.

Definition 6. Let $F$ be a monotone $\Gamma_{I}-$ selection. The $F$-center of imputations, $C I^{F}$, is defined as follows: for all $N \in \mathcal{N}$, all $(N, v) \in \Gamma$ and all $i \in N$,

$$
C I_{i}^{F}(N, v):=v(i)+f_{i}^{v^{I}}\left(N, v(N)-\sum_{i \in N} v(i)\right) .
$$

The difference between $C I^{F}$ and $C I^{f}$ is that, for a given game $(N, v)$, the former distributes the amount $v(N)-\sum_{i \in N} v(i)$ according to a monotone path $f^{v^{I}}$ that depends on $\left(N, v^{I}\right)$, while in $C I^{f}$ this amount is shared by means of a monotone path $f$, that does not depends on the associated game $\left(N, v^{I}\right)$.

Next, we generalize the notion of consistent monotone paths.

Definition 7. A monotone $\Gamma_{I}-$ selection $F$ is said to be consistent if for all $N \in \mathcal{N}$, all $(N, v) \in \Gamma_{I}$, all $N^{\prime} \subset N$ and all $t \in \mathbb{R}$, it holds

$$
f_{j}^{\left(v_{\mid N^{\prime}}\right)^{I}}\left(N^{\prime}, \sum_{i \in N^{\prime}} f_{i}^{v}(N, t)\right)=f_{j}^{v}(N, t) \text { for all } j \in N^{\prime},
$$

being $\left(N^{\prime}, v_{\mid N^{\prime}}\right)$ the subgame of $(N, v)$ associated to $N^{\prime}$.

Now, we have all the tools to characterize the class of single-valued solutions satisfying individual rationality, aggregate monotonicity and projected consistency.

Theorem 7. A single-valued solution $\sigma$ satisfies individual rationality, aggregate monotonicity and projected consistency on $\Gamma$ if and only if there exists a consistent monotone $\Gamma_{I}-$ selection $F$ such that $\sigma=C I^{F}$.

Proof. Let $\sigma$ be a single-valued solution satisfying individual rationality, aggregate monotonicity and projected consistency on $\Gamma$.

Define a monotone $\Gamma_{I}$ - selection $F$ as follows. Take $f \in \mathcal{F}_{\text {mon }}$. For all $N^{\prime} \in \mathcal{N}$ and all $\left(N^{\prime}, v\right) \in \Gamma_{I}$, define

$$
f^{v}(N, t):= \begin{cases}\sigma\left(N, v+t \cdot u_{N}\right)-\sigma(N, v) & \text { if } N=N^{\prime} \\ f(N, t) & \text { if } N \neq N^{\prime}\end{cases}
$$


for all $N \in \mathcal{N}$ and all $t \in \mathbb{R}$.

Let us show that $f^{v} \in \mathcal{F}_{\text {mon. }}$. Clearly, $f^{v}(N, t) \in \mathbb{R}^{N}, \sum_{i \in N} f_{i}^{v}(N, t)=t$ and $f^{v}(N, 0)=(0, \ldots, 0) \in \mathbb{R}^{N}$, for all $N \in \mathcal{N}$ and $t \in \mathbb{R}$. Moreover, if $t^{\prime} \in \mathbb{R}$ is such that $t^{\prime}>t$ and $N=N^{\prime}$, then

$$
\begin{aligned}
f^{v}\left(N, t^{\prime}\right) & =\sigma\left(N, v+t^{\prime} \cdot u_{N}\right)-\sigma(N, v) \\
& \geq \sigma\left(N, v+t \cdot u_{N}\right)-\sigma(N, v) \\
& =f^{v}(N, t)
\end{aligned}
$$

where the inequality follows from the aggregate monotonicity of $\sigma$. If $N \neq N^{\prime}$, we have $f\left(N, t^{\prime}\right) \geq f(N, t)$ since $f \in \mathcal{F}_{\text {mon }}$. Consequently, $f^{v} \in \mathcal{F}_{\text {mon }}$ for all $\left(N^{\prime}, v\right) \in \Gamma_{I}$, and thus $F$ is a monotone $\Gamma_{I}$ - selection.

Let $N \in \mathcal{N}$ and $(N, v) \in \Gamma$. Since $(N, v)$ can be expressed as $v=v^{I}+(v(N)-$ $\left.v^{I}(N)\right) \cdot u_{N}$, it is easy to see that

$$
\sigma(N, v)-\sigma\left(N, v^{I}\right)=f^{v^{I}}\left(N, v(N)-\sum_{i \in N} v(i)\right)
$$

being $f^{v^{I}}$ as defined in (9).

By individual rationality, $\sigma_{i}\left(N, v^{I}\right)=v(i)$ for all $i \in N$, and thus

$$
\sigma_{i}(N, v)=v(i)+f_{i}^{v^{I}}\left(N, v(N)-\sum_{i \in N} v(i)\right) .
$$

Next, we show that $F$ is consistent. Let $N \in \mathcal{N},(N, v) \in \Gamma_{I}, N^{\prime} \subset N$, and $t \in \mathbb{R}$. Define the game $\left(N, v^{\prime}\right)$ as $v^{\prime}=v+t \cdot u_{N}$. Notice that $\left(v^{\prime}\right)^{I}=v$. Let us denote $x=\sigma\left(N, v^{\prime}\right)$. From (10), for all $j \in N^{\prime}$ we have

$$
\sigma_{j}\left(N, v^{\prime}\right)=v^{\prime}(j)+f_{j}^{\left(v^{\prime}\right)^{I}}\left(N, v^{\prime}(N)-\sum_{i \in N} v^{\prime}(i)\right)=v^{\prime}(j)+f_{j}^{v}(N, t)
$$

and

$$
\begin{aligned}
\sigma_{j}\left(N^{\prime}, r_{F, x}^{N^{\prime}}\left(v^{\prime}\right)\right) & =r_{F, x}^{N^{\prime}}\left(v^{\prime}\right)(j)+f_{j}^{\left(r_{F, x}^{N^{\prime}}\left(v^{\prime}\right)\right)^{I}}\left(N^{\prime}, r_{F, x}^{N^{\prime}}\left(v^{\prime}\right)\left(N^{\prime}\right)-\sum_{i \in N^{\prime}} r_{F, x}^{N^{\prime}}\left(v^{\prime}\right)(i)\right) \\
& =v^{\prime}(j)+f_{j}^{\left(v_{\mid N^{\prime}}\right)^{I}}\left(N^{\prime}, \sum_{i \in N^{\prime}} f_{i}^{v}\left(N, v^{\prime}(N)-\sum_{i \in N} v^{\prime}(i)\right)\right) \\
& =v^{\prime}(j)+f_{j}^{\left(v_{\mid N^{\prime}}\right)^{I}}\left(N^{\prime}, \sum_{i \in N^{\prime}} f_{i}^{v}(N, t)\right),
\end{aligned}
$$

where the last before equality comes from (7) in the proof of Theorem 6 , taking into account (10) and the fact that $\left(N^{\prime},\left(r_{F, \sigma}^{N^{\prime}}\left(v^{\prime}\right)\right)^{I}\right)=\left(N^{\prime},\left(v_{\mid N^{\prime}}\right)^{I}\right)$. 
By projected consistency, (11) and (12) must coincide and thus

$$
f_{j}^{v}(N, t)=f_{j}^{\left(v_{\mid N^{\prime}}\right)^{I}}\left(N^{\prime}, \sum_{i \in N^{\prime}} f_{i}^{v}(N, t)\right),
$$

which proves that $F$ is consistent.

Hence, $\sigma=C I^{F}$ being $F$ a consistent monotone $\Gamma_{I}$ - selection.

To show the reverse implication, let $\sigma$ be a single-valued solution on $\Gamma$ such that $\sigma=C I^{F}$, for some consistent monotone $\Gamma_{I}-$ selection $F$. Hence, for all $N \in \mathcal{N}$, all $(N, v) \in \Gamma$ and all $i \in N$ it holds

$$
\sigma_{i}(N, v)=v(i)+f_{i}^{v^{I}}\left(N, v(N)-\sum_{i \in N} v(i)\right) .
$$

From (13), and taking into account that $F$ is consistent, it is not difficult to check individual rationality and projected consistency by using symmetric arguments as in the proof of Theorem 6. Aggregate monotonicity comes from $f^{v^{I}} \in \mathcal{F}_{\text {mon }}$.

The properties in Theorem 7 are independent. ${ }^{12}$

The next corollary comes straightforwardly from the proof of Theorem 7, and extends the characterizations in Theorem 1 and Corollary 2 when we consider aggregate monotonicity instead of either equal surplus division or regular aggregate monotonicity.

Corollary 3. A single-valued solution $\sigma$ satisfies individual rationality and aggregate monotonicity on $\Gamma$ if and only if there exists a monotone $\Gamma_{I}-$ selection $F$ such that $\sigma=C I^{F}$.

\subsection{Impossibility results}

Despite these positive results, unfortunately we find some problems to combine core selection, max consistency and monotonicity properties with respect to the worth of the grand coalition.

\footnotetext{
${ }^{12} E D$ satisfies aggregate monotonicity and projected consistency but not individual rationality, and $\varphi$ satisfies individual rationality and aggregate monotonicity but not projected consistency. Next we introduce a single-valued solution that satisfies individual rationality and projected consistency but not aggregate monotonicity.

Let $g: \mathcal{N} \times \mathbb{R} \rightarrow \bigcup_{N \in \mathcal{N}} \mathbb{R}^{N}$ be a function defined as follows. For all $N \in \mathcal{N}$ and all $t \in \mathbb{R}$, let $S^{N}(t)=\{i \in N|i \geq| t \mid\}$. If $S^{N}(t) \neq \emptyset$, choose $i^{*} \in S^{N}(t)$ to be such that $i^{*} \leq j$, for all $j \in S^{N}(t)$. If $S^{N}(t)=\emptyset$, choose $i^{*} \in N$ to be such that $i^{*} \geq j$, for all $j \in N$. Then, define $g(N, t)=t \cdot e_{\left\{i^{*}\right\}}$.

The function $g$ satisfies conditions $(i)$ and $(i i)$ in the definition of a monotone path (Definition 1 ), but not condition (iii). Moreover, $g$ satisfies expression (3) in the definition of consistent monotone path (Definition 3).

Let $\sigma^{g}$ be the single-valued solution on $\Gamma$ defined as follows: for all $N \in \mathcal{N}$, all $(N, v) \in \Gamma$ and all $i \in N$,

$$
\sigma_{i}^{g}(N, v):=v(i)+g_{i}\left(N, v(N)-\sum_{i \in N} v(i)\right) .
$$

Then, $\sigma^{g}$ satisfies individual rationality and projected consistency but not aggregate monotonicity.
} 
Theorem 8. For $|N| \geq 3$ there is no single-valued solution on $\Gamma$ that satisfies core selection, regular aggregate monotonicity and max consistency.

Proof. Suppose, on the contrary, that there exists a single-valued solution $\sigma$ satisfying core selection, regular aggregate monotonicity and max consistency on $\Gamma$.

Let $\left\{\left(N, v^{k}\right)\right\}_{k \in N}$ be a family of balanced games defined as follows: for each $k \in$ $N=\{1,2,3\}, v^{k}(i)=0$ for all $i \in N, v^{k}(i k)=1$ for all $i \in N \backslash\{k\}, v^{k}(i j)=0$ for all $i, j \in N \backslash\{k\}$, and $v^{k}(N)=3$. For each $k \in N$, let $\left(N, v_{*}^{k}\right)$ be given by $v_{*}^{k}=v^{k}-2 \cdot u_{N}$.

By regular aggregate monotonicity, there exists $f \in \mathcal{F}_{\text {mon }}$ such that $\sigma\left(N, v^{1}\right)-$ $\sigma\left(N, v_{*}^{1}\right)=f\left(N, v^{1}(N)-v_{*}^{1}(N)\right)$. Since $C\left(N, v_{*}^{1}\right)=\{(1,0,0)\}$, by core selection, $\sigma\left(N, v_{*}^{1}\right)=$ $(1,0,0)$ and thus $\sigma\left(N, v^{1}\right)=(1,0,0)+f(N, 2)$. Following similar arguments, and taking into account that $C\left(N, v_{*}^{2}\right)=\{(0,1,0)\}$ and $C\left(N, v_{*}^{3}\right)=\{(0,0,1)\}$, we can write

$$
\sigma\left(N, v^{2}\right)=(0,1,0)+f(N, 2) \text { and } \sigma\left(N, v^{3}\right)=(0,0,1)+f(N, 2) .
$$

We claim that $f_{i}(N, 2)=0$ for all $i \in N$, in contradiction with $\sum_{i \in N} f_{i}(N, 2)=2$.

First, we see that $f_{1}(N, 2)=0$. Since $f \in \mathcal{F}_{\text {mon }}$, we have $f_{1}(N, 2) \geq 0$. Let us denote $x^{k}=\sigma\left(N, v^{k}\right), k \in\{1,2,3\}$, and take $N^{\prime}=\{2,3\}$. Two cases can be distinguished:

- Case 1: $1-f_{1}(N, 2) \leq 0$

In this situation, for all $S \subseteq N^{\prime}$, it holds that $r_{D M, x^{2}}^{N^{\prime}}\left(v^{2}\right)(S)=r_{D M, x^{3}}^{N^{\prime}}\left(v^{3}\right)(S)$. Thus,

$$
\sigma\left(N^{\prime}, r_{D M, x^{2}}^{N^{\prime}}\left(v^{2}\right)\right)=\sigma\left(N^{\prime}, r_{D M, x^{3}}^{N^{\prime}}\left(v^{3}\right)\right)
$$

By max consistency, and considering expression (14), we obtain

$$
\sigma_{2}\left(N^{\prime}, r_{D M, x^{2}}^{N^{\prime}}\left(v^{2}\right)\right)=1+f_{2}(N, 2) \text { and } \sigma_{2}\left(N^{\prime}, r_{D M, x^{3}}^{N^{\prime}}\left(v^{3}\right)\right)=f_{2}(N, 2),
$$

in contradiction with (15).

- Case 2: $1-f_{1}(N, 2)>0$

In this situation, it holds that

$$
\begin{aligned}
& r_{D M, x^{2}}^{N^{\prime}}\left(v^{2}\right)(2)=r_{D M, x^{3}}^{N^{\prime}}\left(v^{3}\right)(3)=1-f_{1}(N, 2) \\
& r_{D M, x^{2}}^{N^{\prime}}\left(v^{2}\right)(3)=r_{D M, x^{3}}^{N^{\prime}}\left(v^{3}\right)(2)=0 \text { and } \\
& r_{D M, x^{2}}^{N^{\prime}}\left(v^{2}\right)\left(N^{\prime}\right)=r_{D M, x^{3}}^{N^{\prime}}\left(v^{3}\right)\left(N^{\prime}\right)=3-f_{1}(N, 2) .
\end{aligned}
$$

For each $k \in N^{\prime}=\{2,3\}$, consider the game $\left(N^{\prime},\left(r_{D M, x^{k}}^{N^{\prime}}\left(v^{k}\right)\right)_{*}\right)$ with characteristic function $\left(r_{D M, x^{k}}^{N^{\prime}}\left(v^{k}\right)\right)_{*}=r_{D M, x^{k}}^{N^{\prime}}\left(v^{k}\right)-2 \cdot u_{N^{\prime}}$. Thus,

$$
\begin{gathered}
C\left(N^{\prime},\left(r_{D M, x^{2}}^{N^{\prime}}\left(v^{2}\right)\right)_{*}\right)=\left\{\left(1-f_{1}(N, 2), 0\right)\right\} \text { and } \\
C\left(N^{\prime},\left(r_{D M, x^{3}}^{N^{\prime}}\left(v^{3}\right)\right)_{*}\right)=\left\{\left(0,1-f_{1}(N, 2)\right)\right\} .
\end{gathered}
$$

By core selection, we have

$$
\sigma\left(N^{\prime},\left(r_{D M, x^{2}}^{N^{\prime}}\left(v^{2}\right)\right)_{*}\right)=\left(1-f_{1}(N, 2), 0\right) \text { and }
$$




$$
\sigma\left(N^{\prime},\left(r_{D M, x^{3}}^{N^{\prime}}\left(v^{3}\right)\right)_{*}\right)=\left(0,1-f_{1}(N, 2)\right) .
$$

By core selection and regular aggregate monotonicity, we can write:

$$
\begin{gathered}
\sigma\left(N^{\prime}, r_{D M, x^{2}}^{N^{\prime}}\left(v^{2}\right)\right)=\left(1-f_{1}(N, 2), 0\right)+f\left(N^{\prime}, 2\right) \text { and } \\
\sigma\left(N^{\prime}, r_{D M, x^{3}}^{N^{\prime}}\left(v^{3}\right)\right)=\left(0,1-f_{1}(N, 2)\right)+f\left(N^{\prime}, 2\right) .
\end{gathered}
$$

Now, applying max consistency and taking into account expression (14), we obtain $f_{3}(N, 2)=f_{3}\left(N^{\prime}, 2\right)$ and $f_{2}(N, 2)=f_{2}\left(N^{\prime}, 2\right)$. But due to $f \in \mathcal{F}_{m o n}$, we have $2=\sum_{i \in N^{\prime}} f_{i}\left(N^{\prime}, 2\right)=f_{2}(N, 2)+f_{3}(N, 2)$, which implies $f_{1}(N, 2)=0$.

Following symmetric arguments for $N^{\prime}=\{1,3\}$ and $N^{\prime}=\{1,2\}$ we obtain $f_{2}(N, 2)=0$ and $f_{3}(N, 2)=0$, respectively, which proves the claim. But then, $\sum_{i \in N} f_{i}(N, 2)=0$, in contradiction with $f \in \mathcal{F}_{\text {mon }}$.

The properties in Theorem 8 are compatible two by two. ${ }^{13}$

Since equal surplus division implies regular aggregate monotonicity, a direct consequence of Theorem 8 is the following.

Corollary 4. For $|N| \geq 3$ there is no single-valued solution on $\Gamma$ that satisfies core selection, equal surplus division and max consistency.

It is not difficult to verify that Theorem 8 remains valid on $\Gamma_{B}$. However, on the domain of convex games core selection, regular aggregate monotonicity and max consistency are compatible.

Let $\pi$ be a permutation on $\mathbb{N}$. The marginal contribution solution relative to $\pi$, denoted by $m c^{\pi}$, is defined as follows: for all $N \in \mathcal{N}$, all $(N, v) \in \Gamma$ and all $i \in N$

$$
m c_{i}^{\pi}:=v(\{j \in N \mid \pi(j) \leq \pi(i)\})-v(\{j \in N \mid \pi(j)<\pi(i)\}) .
$$

It is easy to check that $m c^{\pi}$ satisfies regular aggregate monotonicity according to $f^{\pi} \in$ $\mathcal{F}_{\text {mon }}$ as defined in Section 2. Moreover, it is well-known that $m c^{\pi}$ satisfies core selection and max consistency on $\Gamma_{C}$ (see, for instance, Hokari and van Gellekom, 2002).

By contrast, the next example shows that core selection, equal surplus division and max consistency are incompatible on the domain of convex games for $|N| \geq 3$.

Example 1. Suppose, on the contrary, that there exists a single-valued solution $\sigma$ satisfying core selection, equal surplus division and max consistency on $\Gamma_{C}$.

Let $(N, v)$ be a convex game with set of players $N=\{1,2,3\}$ and characteristic function as follows: $v(12)=v(123)=1$ and $v(S)=0$ otherwise. Note that for all $x \in C(N, v), x_{3}=0$. Let us denote $x=\sigma(N, v)$. By core selection, $x_{3}=0$. Take $N^{\prime}=\{1,2\}$ and consider the max reduced game $\left(N^{\prime}, r_{D M, x}^{N^{\prime}}(v)\right)$, being $r_{D M, x}^{N^{\prime}}(v)(1)=$ $r_{D M, x}^{N^{\prime}}(v)(2)=0$ and $r_{D M, x}^{N^{\prime}}(v)\left(N^{\prime}\right)=1$. Clearly, $\left(N^{\prime}, r_{D M, x}^{N^{\prime}}(v)\right) \in \Gamma_{C}$. Now define the convex game $\left(N^{\prime},\left(r_{D M, x}^{N^{\prime}}(v)\right)_{*}\right)$ as $\left(r_{D M, x}^{N^{\prime}}(v)\right)_{*}=r_{D M, x}^{N^{\prime}}(v)-u_{N^{\prime}}$. By core selection,

\footnotetext{
${ }^{13} \nu_{*}$ satisfies core selection and $\max$ consistency, $\bar{\nu}_{*}$ satisfies core selection and equal surplus division and ED satisfies equal surplus division and max consistency.
} 
$\sigma\left(N^{\prime},\left(r_{D M, x}^{N^{\prime}}(v)\right)_{*}\right)=(0,0)$. Thus, by equal surplus division $\sigma\left(N^{\prime}, r_{D M, x}^{N^{\prime}}(v)\right)=\left(\frac{1}{2}, \frac{1}{2}\right)$ and, by max consistency, $\sigma(N, v)=\left(\frac{1}{2}, \frac{1}{2}, 0\right)$.

Now, let $\left(N, v^{\prime}\right)$ be the convex game given by $v^{\prime}=v+3 \cdot u_{N}$. By equal surplus division, $\sigma\left(N, v^{\prime}\right)=\left(\frac{3}{2}, \frac{3}{2}, 1\right)=y \in C\left(N, v^{\prime}\right)$. Take $N^{\prime}=\{1,3\}$. Then, $\left(N^{\prime}, r_{D M, y}^{N^{\prime}}\left(v^{\prime}\right)\right) \in$ $\Gamma_{C}$ with $r_{D M, y}^{N^{\prime}}\left(v^{\prime}\right)(1)=r_{D M, y}^{N^{\prime}}\left(v^{\prime}\right)(3)=0$ and $r_{D M, y}^{N^{\prime}}\left(v^{\prime}\right)\left(N^{\prime}\right)=\frac{5}{2}$. Define the convex game $\left(N^{\prime},\left(r_{D M, y}^{N^{\prime}}\left(v^{\prime}\right)\right)_{*}\right)$ as $\left(r_{D M, y}^{N^{\prime}}\left(v^{\prime}\right)\right)_{*}=r_{D M, y}^{N^{\prime}}\left(v^{\prime}\right)-\frac{5}{2} \cdot u_{N^{\prime}}$. By core selection, $\sigma\left(N^{\prime},\left(r_{D M, y}^{N^{\prime}}\left(v^{\prime}\right)\right)_{*}\right)=(0,0)$ and, by equal surplus division, $\sigma\left(N^{\prime}, r_{D M, y}^{N^{\prime}}\left(v^{\prime}\right)\right)=$ $\left(\frac{5}{4}, \frac{5}{4}\right) \neq\left(\frac{3}{2}, 1\right)$, which contradicts max consistency.

Table 4 summarizes the (in)compatibilities of properties of rationality combined with properties of consistency and properties of monotonicity for single-valued solutions on $\Gamma$.

Table 4. (In)compatibilities of rationality, consistency and monotonicity on $\Gamma$

\begin{tabular}{l|cccc} 
& ESD & RAM & AM \\
\hline IR + P-CON & $C I$ & $C I^{f}($ Th. 6) & $C I^{F}$ (Th. 7) \\
CS + M-CON & $\times($ Th. 8) & $\times($ Th. 8) & Open
\end{tabular}

\section{Final remarks}

We have studied the compatibility between rationality properties (core selection and individual rationality), properties of monotonicity with respect to the worth of the grand coalition (equal surplus division, regular aggregate monotonicity and aggregate monotonicity) together with consistency properties ( max consistency, complement consistency and projected consistency). Although our results are stated on the domain of all games $\Gamma$, some of them remain valid on the domain of balanced games $\Gamma_{B}$ or on the domain of essential games $\Gamma_{E}$. In particular, Theorems 1, 2, 3, 6 and 7, and Corollaries 1, 2 and 3 hold on $\Gamma_{E}$. Moreover, Theorems 4 and 5 , and Corollary 4 hold on $\Gamma_{B}$. It is worth to stress that for games with at most two players, $S h$ satisfies all the properties we have worked with.

Theorem 8 states that core selection, regular aggregate monotonicity and max consistency are incompatible on the domain of all games and, as we have commented before, also in the domain of balanced games. Thus, core selection and max consistency can only be combined with non-regular monotonicity properties. On the domain of balanced games (and also on the domain of all games) with at most three players, core selection, aggregate monotonicity and max consistency are compatible by means of $\nu_{*}$ (Housman and Clark, 1998). However, it is still an open question if core selection, aggregate monotonicity and max consistency are compatible on the domain of balanced games with at least four players.

Finally, Corollary 1 opens another interesting question: which kind of consistency principles are compatibles with core selection and individual rationality together? 


\section{References}

[1] Arin, J. and I. Katsev (2014) The SD-prenucleolus for TU games. Mathematical Methods of Operations Research, 80 : 307-327.

[2] Béal, S., E. Rémila and P. Solal (2014) Axioms of invariance for TU-games. International Journal of Game Theory, DOI 10.1007/s00182-014-0458-2.

[3] Calleja, P., C. Rafels and S. Tijs (2012) Aggregate monotonic stable single-valued solutions for cooperative games. International Journal of Game Theory, 41: 899-913.

[4] Casajus A, and F. Huettner (2014) Null, nullifying, or dummifying players: The difference between the Shapley value, the equal division value, and the equal surplus division value. Economics Letters, 12: 167-169.

[5] Chun, Y. and B. Park (2012) Population solidarity, population fair-ranking, and the egalitarian value. International Journal of Game Theory, 41: 255-270.

[6] Davis, M. and M. Maschler (1965) The kernel of a cooperative game. Naval Research Logistics Quarterly, 12: 223-259.

[7] Dutta, B. and D. Ray (1989) A concept of egalitarianism under participation constraints. Econometrica, 57: 615-635.

[8] Driessen, T. (1991) A survey of consistency properties in cooperative game theory. SIAM Rev 33: 43-59.

[9] Driessen, T. and Y. Funaki (1991). Coincidence of and collinearity between game theoretic solutions. OR Spektrum 13: 15-30.

[10] Funaki, Y. (1998) Dual axiomatizations of solutions of cooperative games. Mimeo.

[11] Gillies D.B (1953) Some theorems on n-person games, Ph.D.Dissertation, Princeton University Press, Princeton.

[12] Grotte, J. (1970). Computation of and observations on the nucleolus, the normalized nucleolus and the central games. PhD thesis, Cornell University, Ithaca

[13] Hokari, T. (2002). Monotone-path Dutta-Ray solutions on convex games. Social Choice and Welfare, 19: 825-844.

[14] Hokari, T. and A. Gellekom (2002). Population monotonicity and consistency in convex games: Some logical relations. International Journal of Game Theory, 31: 593607.

[15] Housman, D. and L. Clark (1998) Core and monotonic allocations methods. International Journal of Game Theory, 27: 611-616.

[16] Hwang, Y. (2013) On the core: complement-reduced game and max-reduced game. International Journal of Game Theory, 42: 339-355. 
[17] Llerena, F. and C. Rafels (2007). Convex decomposition of games and axiomatizations of the core and the D-core. International Journal of Game Theory, 35: 603-615.

[18] Megiddo, N. (1974) On the nonmonotonicity of the bargaining set, the kernel and the nucleolus of a game. SIAM J. Appl Math 27: 355-358.

[19] Moulin, H. (1985) The separability axiom and equal sharing methods. Journal of Economic Theory, 36: 120-148.

[20] Peleg, B. (1986) On the reduced game property and its converse. International Journal of Game Theory, 15: 187-200.

[21] Potters, J. (1991) An axiomatization of the nucleolus. International Journal of Game Theory, 19: 365-373.

[22] Shapley, L.S. (1953) A value for n-person games. In: Kuhn HW, Tucker NW (eds.) Contributions to the theory of games. Princeton University Press, Princeton, 307-317.

[23] Schmeidler, D. (1969) The nucleolus of a characteristic function game. SIAM Journal Appl Math, 17: 1163-1170.

[24] Tadenuma, K. (1992) Reduced games, consistency, and the core. International Journal of Game Theory, 20: 325-334.

[25] Thomson, W. (2003) Consistent allocation rules. Mimeo, University of Rochester.

[26] van den Brink, R. (2007) Null or nullifying players: the difference between the Shapley value and equal division solutions. Journal of Economic Theory, 136: 767-775

[27] van den Brink, R., Y. Chun, Y. Funaki and B. Park (2012) Consistency, population solidarity, and egalitarian solutions for TU-games. Tinbergen Discussion Paper 2012136/II, Tinbergen Institute and VU Amsterdam. 